\title{
Pattern of Distant Metastases and Promising Prognostic Factors in Bladder Cancer: A Population-Based Study
}

\author{
Yanbo Xu ${ }^{1}$, Hong Liu ${ }^{2}$, Yue Liu ${ }^{1}$, Qihua $\mathrm{Cao}^{1}$, Dong $\mathrm{Xu}^{3,}$ * \\ ${ }^{1}$ Department of Surgical Oncology, the Second Affiliated Hospital of Zhejiang University, Hangzhou, China \\ ${ }^{2}$ Department of Medical Oncology, the Second Affiliated Hospital of Zhejiang University, Hangzhou, China \\ ${ }^{3}$ Department of Surgical Oncology and Cancer Institute, the Second Affiliated Hospital of Zhejiang University, Hangzhou, China
}

Email address:

xudongzju@zju.edu.cn (Dong Xu)

${ }^{*}$ Corresponding author

\section{To cite this article:}

Yanbo Xu, Hong Liu, Yue Liu, Qihua Cao, Dong Xu. Pattern of Distant Metastases and Promising Prognostic Factors in Bladder Cancer: A Population-Based Study. Journal of Cancer Treatment and Research. Vol. 8, No. 1, 2020, pp. 1-9. doi: 10.11648/j.jctr.20200801.11

Received: December 19, 2019; Accepted: December 30, 2019; Published: January 8, 2020

\begin{abstract}
The aims of this study were to evaluate the incidence and survival rates of distant metastases in newly diagnosed bladder cancer patients, and to investigate the risk and prognostic factors as well. The Surveillance, Epidemiology, and End Results (SEER) database was analyzed for the incidence and survival rates of patients diagnosed with bladder cancer between 2010-2015. Multivariate logistic regression analyses identified risk factors for distant metastases. The survival rates were calculated by Kaplan Meier analysis. Cox regression and Fine and Grey's model analyses were used to estimate prognostic factors associated with metastatic bladder cancers. Totally, there were 65541 patients with bladder cancer obtained from the SEER database, and 2812 were diagnosed with distant metastases on initial diagnosis (4.3\%). Metastatic distribution information was concluded liver, lung, bone, brain and others. Of these, 1029 patients were bone metastases, 988 patients were lung metastases, 687 patients were liver metastases and 97 patients were brain metastases. As a result, younger age, unmarried social status, Black race, non-transitional cell carcinoma subtypes, poor differentiated grade, increased tumor stage and lymph node stage, absence of surgery to primary sites or unknown were all significantly associated with early distant metastases by logistic regression. Multivariate Cox regression analysis showed that older age, female, unmarried social status, non-TCC subtypes, increased number of metastatic sites and absence of surgery to primary sites were significantly associated with reduced overall survival. These findings in metastatic bladder cancer may help to arrange thorough evaluation and then make individualized treatment plan.
\end{abstract}

Keywords: Bladder Cancer, Metastasis, Incidence, Prognosis

\section{Introduction}

Bladder cancer is the forth-most common malignancy in male, with estimated more than 80,000 new diagnosed cases and more than 17,000 deaths annually in the United States [1]. The majority of patients with bladder cancer is diagnosed at early stage (no muscle invasive), with a 5-year overall survival over $80 \%$ [2]. However, because of the high recurrence and metastases, it is hard to cure [3]. When there is metastatic progression, the 5-year survival rate dramatically decreases to $15 \%$ [2]. As it was previously reported, unfortunately, about $10-15 \%$ of patients with bladder cancer were already metastatic at initial diagnosis [3-5]. Metastatic bladder cancer patients usually have a poor prognosis, with only a few months survival time, owing to the invasion to the muscle layer $[6,7]$. Different metastatic sites may represent different biological behavior and prognosis and subsequently, requiring different therapeutic approaches [8, 9]. However, little is known on the metastatic distribution of bladder cancer. Therefore, the aims of this study are to analyze incidence and risk factors with different metastases at initial diagnosis of bladder cancer patients, as well as survival rates and prognostic factors. 


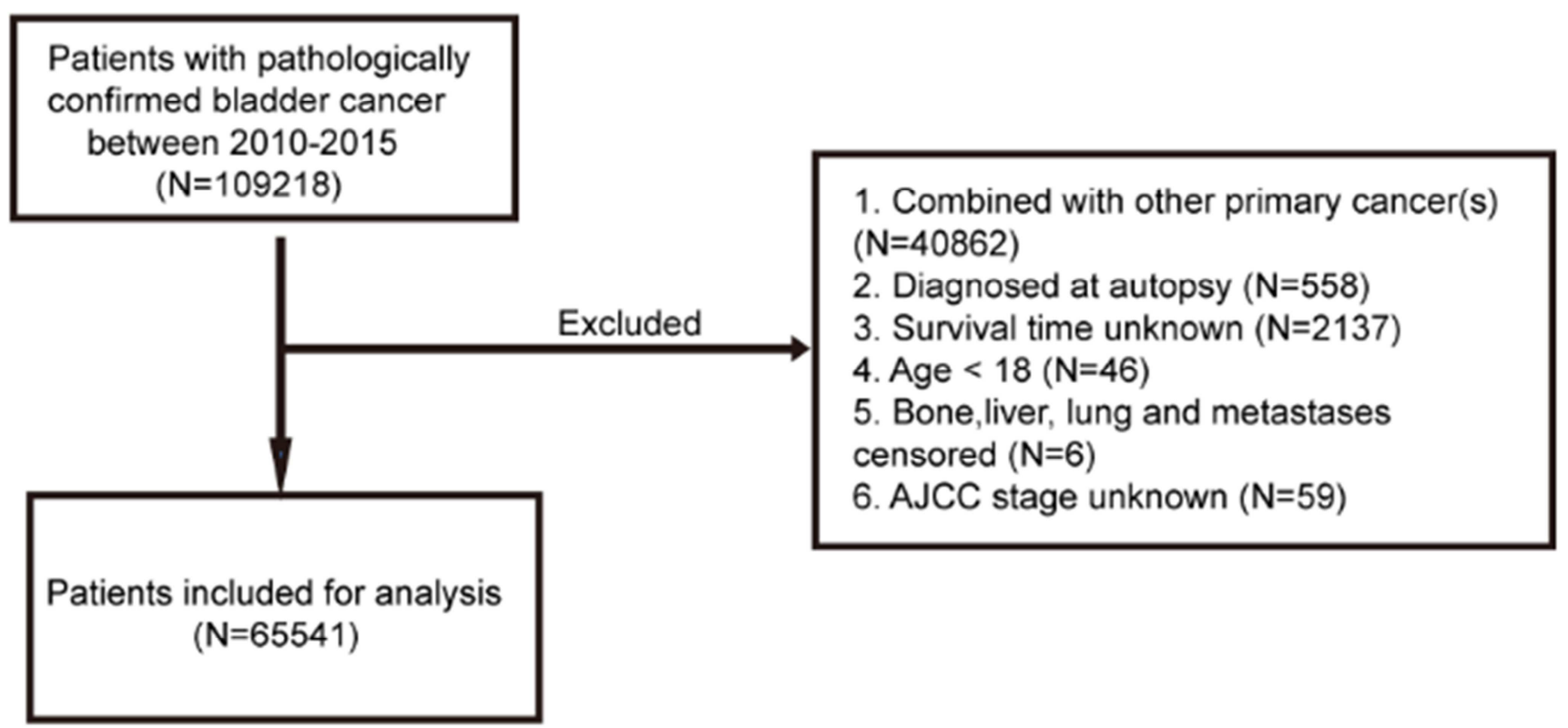

Figure 1. Flowchart of patient selection.

\section{Materials and Methods}

\subsection{Cohort Population}

People diagnosed with bladder cancer between 2010 and 2015 were obtained from the SEER database. Totally, we identified 65541 patients 18 years or older, with single primary malignancies, clear metastatic status and specific survival time, excluding those who were diagnosed at autopsy. The detailed inclusion and exclusion criterions were showed in Figure 1. Data extracted for each patient included: age at diagnosis, sex, marital status, race, histology, grade, Tumor stage (T stage) and Lymph node stage ( $\mathrm{N}$ stage), surgery to the primary tumor, cause of death and survival months. Metastatic bladder cancer patients were categorized according to the site of metastases: bone metastases, brain metastases, liver metastases, lung metastases and others (having distant metastasis but the exact site was unknown). Race/ethnicity was categorized as white, black, or others according to the SEER database. Informed consent was not required because the data in this study did not contain human subjects or personal information.

\subsection{Statistical Analysis}

Categorical variables were reported as whole numbers and proportions. The baseline characteristics of categorical variables were calculated by Chi-square test and Fisher's exact test. Multivariable logistic regression was used to identify whether age, sex, marital status, race, histology, grade, tumor stage, lymph node stage and surgery to the primary tumor were related to the presence of distant metastases in newly diagnosed bladder cancer. Overall survival (OS) was defined from the time of diagnosis to any cause of death. The OS was generated using the Kaplan-Meier method, and differences were examined using the log-rank test. The associations of relevant clinicopathological variables with prognostic factors were assessed using Cox regression and Fine and Grey's model. Odds ratio (OR), Hazard ratio (HR) and $95 \%$ confidential interval $(\mathrm{CI})$ were reported. A two-sided p-value $<0.05$ was considered statistically significant. All of the statistical analyses were completed using $\mathrm{R}$ (version 3.4.3; R Foundation).

\section{Results}

\subsection{Patient Characteristics}

A total of 65541 patients with bladder cancer were included in this study according to the criteria described above. The demographic and clinicopathological characteristics of patients with distant metastases are summarized in Table 1. $44416(67.768 \%)$ patients were $\geq 65$ years old, 48869 $(74.562 \%)$ were male, $36892(56.289 \%)$ had been married, $57123(87.156 \%)$ were of white race, $62002(94.600 \%)$ were transitional cell cancer among most of patients (29379, $44.825 \%$ ) presented with higher grade (grade III and IV) tumors. Over half (75.954\%) of patients with bladder cancer were found to be early stage (stage T0, Tis, Ta and T1). Nearly all the patients $(92.504 \%)$ were found no regional lymph node metastasis. $60211(91.868 \%)$ patients included were underwent surgery to primary sites.

\subsection{Metastasis}

Chi-square test and Fisher's exact test showed that the following factors were all significantly associated with the presence of metastases among patients with bladder cancer: age $(p=0.02)$, marital status, sex, marital status, race, pathology, grade, $\mathrm{T}$ and $\mathrm{N}$ stages, surgery to primary sites $(\mathrm{p}<0.001)$ (Table 1). 
Table 1. Clinicopathological features and incidence proportions of patients with bladder cancer by different metastatic sites.

\begin{tabular}{|c|c|c|c|c|c|c|c|c|c|}
\hline & \multicolumn{3}{|l|}{ Metastases } & \multicolumn{3}{|c|}{ Bone metastases } & \multicolumn{3}{|c|}{ Brain metastases } \\
\hline & Yes N (\%) & No N (\%) & $p$ & Yes N (\%) & No N (\%) & $p$ & Yes N (\%) & No N (\%) & $p$ \\
\hline Total & $2812(4.3)$ & $62729(95.7)$ & & $1029(1.6)$ & $64512(98.4)$ & & $97(0.1)$ & $65444(99.9)$ & \\
\hline Age & & & 0.020 & & & $<0.001$ & & & 0.015 \\
\hline$<65$ years & 963 (1.5) & $20162(30.8)$ & & $392(0.6)$ & $20733(31.6)$ & & $43(0.1)$ & $21082(32.2)$ & \\
\hline$\geq 65$ years & $1849(2.8)$ & $42567(64.9)$ & & $637(1.0)$ & $43779(66.8)$ & & $54(0.1)$ & $44362(67.7)$ & \\
\hline Sex & & & $<0.001$ & & & 0.705 & & & 0.510 \\
\hline Female & $832(1.3)$ & $15840(24.2)$ & & $256(0.4)$ & $16416(25.0)$ & & $28(0.0)$ & $16644(25.4)$ & \\
\hline Male & $1980(3.0)$ & 46889 (71.5) & & 773 (1.2) & $48096(73.4)$ & & $69(0.1)$ & $48800(74.5)$ & \\
\hline Marital status & & & $<0.001$ & & & $<0.001$ & & & $<0.001$ \\
\hline Married & $1297(2.0)$ & $35595(54.3)$ & & $464(0.7)$ & $36428(55.6)$ & & $40(0.1)$ & $36852(56.2)$ & \\
\hline Not married & $1388(2.1)$ & $21439(32.7)$ & & $513(0.8)$ & $22314(34.0)$ & & $54(0.1)$ & $22773(34.7)$ & \\
\hline Unknown & $127(0.2)$ & $5695(8.7)$ & & $52(0.1)$ & $5770(8.8)$ & & $3(0.0)$ & $5819(8.9)$ & \\
\hline Race & & & $<0.001$ & & & $<0.001$ & & & 0.490 \\
\hline Black & $300(0.5)$ & $3571(5.4)$ & & $102(0.2)$ & $3769(5.8)$ & & $7(0.0)$ & $3864(5.9)$ & \\
\hline White & $2372(3.6)$ & $54751(83.5)$ & & $876(1.3)$ & $56247(85.8)$ & & $86(0.1)$ & $57037(87.0)$ & \\
\hline Others $^{\mathrm{a}}$ & $137(0.2)$ & 3061 (4.7) & & $50(0.1)$ & $3148(4.8)$ & & $4(0.0)$ & $3194(4.9)$ & \\
\hline Unknown & $3(0.0)$ & $1346(2.1)$ & & $1(0.0)$ & $1348(2.1)$ & & $0(0)$ & $1349(2.1)$ & \\
\hline Pathology & & & $<0.001$ & & & $<0.001$ & & & $<0.001$ \\
\hline Not TCC & $684(1.0)$ & $2855(4.4)$ & & $236(0.4)$ & $3303(5.0)$ & & $25(0.0)$ & $3514(5.4)$ & \\
\hline TCC & $2128(3.2)$ & $59874(91.4)$ & & 793 (1.2) & $61209(93.4)$ & & $72(0.1)$ & $61930(94.5)$ & \\
\hline Grade $^{b}$ & & & $<0.001$ & & & $<0.001$ & & & $<0.001$ \\
\hline $\mathrm{I} \sim \mathrm{II}$ & $121(0.2)$ & 20029 (30.6) & & $37(0.1)$ & $20113(30.7)$ & & $4(0.0)$ & $20146(30.7)$ & \\
\hline III & $626(1.0)$ & $7987(12.2)$ & & $216(0.3)$ & 8397 (12.8) & & $26(0.0)$ & 8587 (13.1) & \\
\hline IV & $1297(2.0)$ & $19469(29.7)$ & & $468(0.7)$ & $20298(31.0)$ & & $32(0.0)$ & 20734 (31.6) & \\
\hline Unknown & $768(1.2)$ & $15244(23.3)$ & & $308(0.5)$ & $15704(24.0)$ & & $35(0.1)$ & $15977(24.4)$ & \\
\hline Tumor stage & & & $<0.001$ & & & $<0.001$ & & & $<0.001$ \\
\hline T0, Tis, Ta & $33(0.1)$ & $34499(52.6)$ & & $14(0.0)$ & $34518(52.7)$ & & $1(0.0)$ & $34531(52.7)$ & \\
\hline $\mathrm{T} 1$ & $428(0.7)$ & $14821(22.6)$ & & $159(0.2)$ & $15090(23.0)$ & & $12(0.0)$ & $15237(23.2)$ & \\
\hline $\mathrm{T} 2$ & $1033(1.6)$ & 8045 (12.3) & & $390(0.6)$ & $8688(13.3)$ & & $37(0.1)$ & $9041(13.8)$ & \\
\hline $\mathrm{T} 3$ & $250(0.3)$ & $2220(3.4)$ & & $74(0.1)$ & $2396(3.7)$ & & $7(0.0)$ & $2463(3.8)$ & \\
\hline $\mathrm{T} 4$ & $514(0.8)$ & $1566(2.4)$ & & $170(0.3)$ & $1910(2.9)$ & & $9(0.0)$ & $2071(3.2)$ & \\
\hline Tx & $554(0.8)$ & $1578(2.4)$ & & $222(0.3)$ & $1910(2.9)$ & & $31(0.0)$ & $2101(3.2)$ & \\
\hline Lymph node stage & & & $<0.001$ & & & $<0.001$ & & & $<0.001$ \\
\hline N0 & $1430(2.2)$ & $59198(90.3)$ & & $557(0.9)$ & $60071(91.7)$ & & $54(0.1)$ & $60574(92.4)$ & \\
\hline N1 & $279(0.4)$ & $814(1.2)$ & & $97(0.1)$ & $996(1.5)$ & & $6(0.0)$ & $1087(1.7)$ & \\
\hline $\mathrm{N} 2$ & $515(0.8)$ & $908(1.4)$ & & $167(0.3)$ & $1256(1.9)$ & & $11(0.0)$ & $1412(2.2)$ & \\
\hline N3 & $178(0.3)$ & $190(0.3)$ & & $58(0.1)$ & $310(0.5)$ & & $6(0.0)$ & $362(0.6)$ & \\
\hline $\mathrm{Nx}$ & $410(0.6)$ & $1619(2.5)$ & & $150(0.2)$ & $1879(2.9)$ & & $20(0.0)$ & $2009(3.1)$ & \\
\hline Surgery of primary site & & & $<0.001$ & & & $<0.001$ & & & $<0.001$ \\
\hline Yes & $1968(3.0)$ & $58243(88.9)$ & & $702(1.1)$ & $59509(90.8)$ & & $59(0.1)$ & $60152(91.8)$ & \\
\hline No/unknown & $844(1.3)$ & $4486(6.8)$ & & $327(0.5)$ & $5003(7.6)$ & & $38(0.1)$ & $5292(8.1)$ & \\
\hline
\end{tabular}

Table 1. Continued.

\begin{tabular}{|c|c|c|c|c|c|c|}
\hline & \multicolumn{3}{|c|}{ Liver metastases } & \multicolumn{3}{|c|}{ Lung metastases } \\
\hline & Yes N (\%) & No N (\%) & $p$ & Yes N (\%) & No N (\%) & $p$ \\
\hline Total & $687(1.0)$ & $64854(99.0)$ & & $988(1.5)$ & $64553(98.5)$ & \\
\hline Age & & & 0.508 & & & 1 \\
\hline$<65$ years & $230(0.4)$ & $20895(31.9)$ & & $318(0.5)$ & $20807(31.7)$ & $<0.001$ \\
\hline$\geq 65$ years & $457(0.7)$ & $43959(67.1)$ & & $670(1.0)$ & $43746(66.7)$ & \\
\hline Sex & & & 0.262 & & & $<0.001$ \\
\hline Female & $188(0.3)$ & $16484(25.2)$ & & $308(0.5)$ & $16364(25.0)$ & \\
\hline Male & $499(0.8)$ & $48370(73.8)$ & & $680(1.0)$ & $48189(73.5)$ & \\
\hline Marital status & & & $<0.001$ & & & $<0.001$ \\
\hline
\end{tabular}




\begin{tabular}{|c|c|c|c|c|c|c|}
\hline & \multicolumn{3}{|c|}{ Liver metastases } & \multicolumn{3}{|c|}{ Lung metastases } \\
\hline & Yes N (\%) & No N (\%) & $p$ & Yes N (\%) & No N (\%) & $p$ \\
\hline Married & $319(0.5)$ & $36573(55.8)$ & & $440(0.7)$ & $36452(55.6)$ & \\
\hline Not married & $336(0.5)$ & $22491(34.3)$ & & $505(0.8)$ & $22322(34.1)$ & \\
\hline Unknown & $32(0.0)$ & $5790(8.8)$ & & $43(0.1)$ & $5779(8.8)$ & \\
\hline Race & & & $<0.001$ & & & $<0.001$ \\
\hline Black & $66(0.1)$ & $3805(5.8)$ & & $120(0.2)$ & $3751(5.7)$ & \\
\hline White & $586(0.9)$ & $56537(86.3)$ & & $824(1.3)$ & $56299(85.9)$ & \\
\hline Others $^{\mathrm{a}}$ & $34(0.1)$ & $3164(4.8)$ & & $44(0.1)$ & $3154(4.8)$ & \\
\hline Unknown & $1(0.0)$ & $1348(2.1)$ & & $0(0)$ & $1349(2.1)$ & \\
\hline Pathology & & & $<0.001$ & & & $<0.001$ \\
\hline Not TCC & $234(0.4)$ & $3305(5.0)$ & & $234(0.4)$ & $3305(5.0)$ & \\
\hline TCC & $453(0.7)$ & $61549(93.9)$ & & $754(1.2)$ & $61248(93.5)$ & \\
\hline Grade $^{b}$ & & & $<0.001$ & & & $<0.001$ \\
\hline $\mathrm{I} \sim \mathrm{II}$ & $26(0.0)$ & $20124(30.7)$ & & $54(0.1)$ & $20096(30.7)$ & \\
\hline III & $150(0.2)$ & 8463 (12.9) & & $223(0.3)$ & $8390(12.8)$ & \\
\hline IV & $268(0.4)$ & $20498(31.3)$ & & $444(0.7)$ & $20322(31.0)$ & \\
\hline Unknown & $243(0.4)$ & $15769(24.1)$ & & $267(0.4)$ & $15745(24.0)$ & \\
\hline Tumor stage & & & $<0.001$ & & & $<0.001$ \\
\hline T0, Tis, Ta & $13(0.0)$ & $34519(52.7)$ & & $13(0.0)$ & $34519(52.7)$ & \\
\hline $\mathrm{T} 1$ & $114(0.2)$ & $15135(23.1)$ & & $158(0.2)$ & $15091(23.0)$ & \\
\hline $\mathrm{T} 2$ & $213(0.3)$ & 8865 (13.5) & & $366(0.6)$ & $8712(13.3)$ & \\
\hline $\mathrm{T} 3$ & $46(0.1)$ & $2424(3.7)$ & & $74(0.1)$ & $2396(3.7)$ & \\
\hline $\mathrm{T} 4$ & $115(0.2)$ & $1965(3.0)$ & & $171(0.3)$ & 1909 (2.9) & \\
\hline Tx & $186(0.3)$ & $1946(3.0)$ & & $206(0.3)$ & $1926(2.9)$ & \\
\hline Lymph node stage & & & $<0.001$ & & & $<0.001$ \\
\hline N0 & $352(0.5)$ & $60276(92.0)$ & & $525(0.8)$ & 60103 (91.7) & \\
\hline N1 & $69(0.1)$ & $1024(1.6)$ & & $102(0.2)$ & $991(1.5)$ & \\
\hline $\mathrm{N} 2$ & $117(0.2)$ & $1306(2.0)$ & & $164(0.3)$ & $1259(1.9)$ & \\
\hline $\mathrm{N} 3$ & $35(0.1)$ & $333(0.5)$ & & $43(0.1)$ & $325(0.5)$ & \\
\hline Nx & $114(0.2)$ & $1915(2.9)$ & & $154(0.2)$ & $1875(2.9)$ & \\
\hline Surgery of primary site & & & $<0.001$ & & & $<0.001$ \\
\hline Yes & $424(0.6)$ & $59787(91.2)$ & & $693(1.1)$ & $59518(90.8)$ & \\
\hline No/unknown & $263(0.4)$ & $5067(7.7)$ & & $295(0.5)$ & $5035(7.7)$ & \\
\hline
\end{tabular}

$\mathrm{N}=$ number; $\mathrm{CI}=$ confidence interval; $\mathrm{TCC}=$ transitional cell carcinoma.

a. Including American Indian/AK Native, Asian/Pacific Islander.

b. Grade: I, well differentiated; II, moderately differentiated; III, poorly differentiated; IV, undifferentiated.

Numbers do not add up to $100 \%$ due to round-off errors.

Then on multivariable logistic regression (Figure 2), unmarried (vs. married; odds ratio [OR], 1.173; 95\% CI, 1.071-1.284; $\mathrm{P}=0.001$ ), grade III (vs. grade I-II; OR, 2.287; 95\% CI, 1.850-2.847; $\mathrm{P}<0.001$ ) and grade IV (grade I-II; OR, 1.992; 95\% CI, 1.625-2.462; $\mathrm{P}<0.001$ ), stage T2 (vs. stage T1; OR, 3.433; 95\%CI, 3.041-3.882; $\mathrm{P}<0.001$ ), stage T3 (vs. stage T1; OR, 1.615; 95\% CI, 1.346-1.933; $\mathrm{P}<0.001$ ), and stage T4 (vs. stage T1; OR, 3.991; 95\%CI, 3.408-4.673; $\mathrm{P}<0.001$ ), stage $\mathrm{N} 1$ (vs. stage N0; OR, 3.952; 95\% CI, 3.639-4.626; $\mathrm{P}<0.001$ ), stage N2 (vs. stage N0; OR, 6.372; $95 \% \mathrm{CI}, 5.556-7.305 ; \mathrm{P}<0.001$ ), and stage $\mathrm{N} 3$ (vs. stage $\mathrm{N} 0$; OR, 11.482; 95\% CI, 9.144-14.415; $\mathrm{P}<0.001)$ and absence of surgery to primary sites or unknown (vs. surgery; OR, 3.580; $95 \%$ CI, 3.109-4.120; $\mathrm{P}<0.001)$ were associated with significantly greater odds of having metastases at diagnosis. Sex was not associated with a risk of metastasis in the multivariable analysis. $\geq 65$ years old (OR, $0.818 ; 95 \% \mathrm{CI}$, 0.746-0.898; $\mathrm{P}<0.001)$, White race $(\mathrm{OR}, 0.848 ; 95 \% \mathrm{CI}$, $0.735-0.981 ; \mathrm{P}<0.001)$ and other race $(\mathrm{OR}, 0.778 ; 95 \% \mathrm{CI}$, 0.614-0.983; $\mathrm{P}<0.001)$, TCC histology (OR, $0.625 ; 95 \% \mathrm{CI}$, 0.555-0.706; $\mathrm{P}<0.001)$, and T0, Tis, Ta stages (OR, 0.046; $95 \%$ CI, $0.032-0.065 ; \quad \mathrm{P}<0.001)$ were associated with marginally lower odds of metastasis at diagnosis. Significant results were presented in Figure 2. 


\section{Multivariable Logistic Regression}

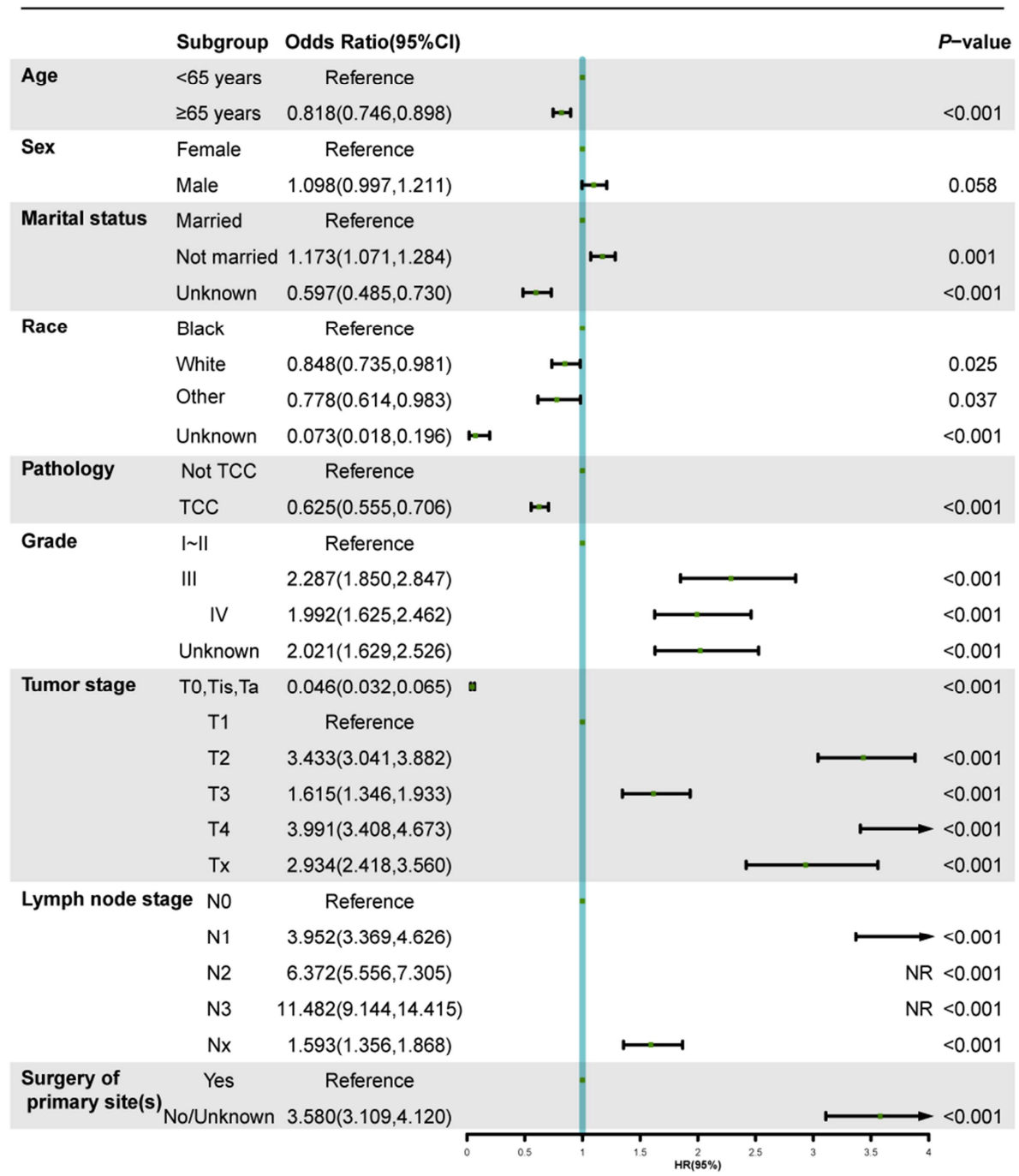

Abbreviation: TCC, transitional cell carcinoma.

Figure 2. Multivariate logistic regression for patients of bladder cancer with distant metastases at initial diagnosis.

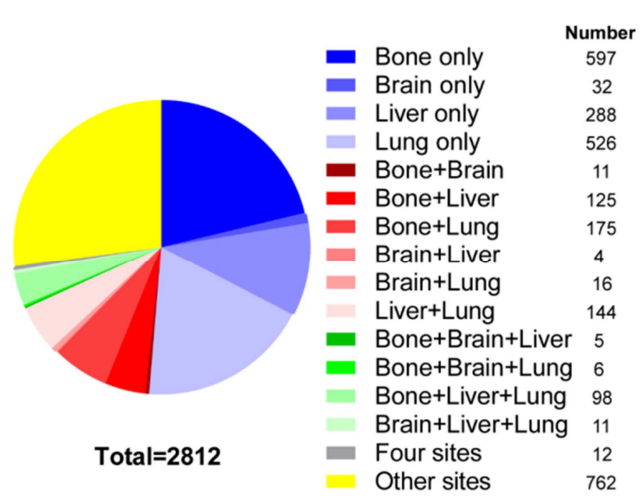

Figure 3. Absolute numbers and relative rates of single and combined metastases in bladder cancer at initial diagnosis.

Among the 65541 patients with bladder cancer, there were $2812(4.3 \%)$ patents with distant metastases at initial diagnosis. The characteristics and distribution of different metastatic sites were presented in Table 1 . It is a great pity that the metastatic information in SEER database just involved in liver, lung, bone and brain metastasis.

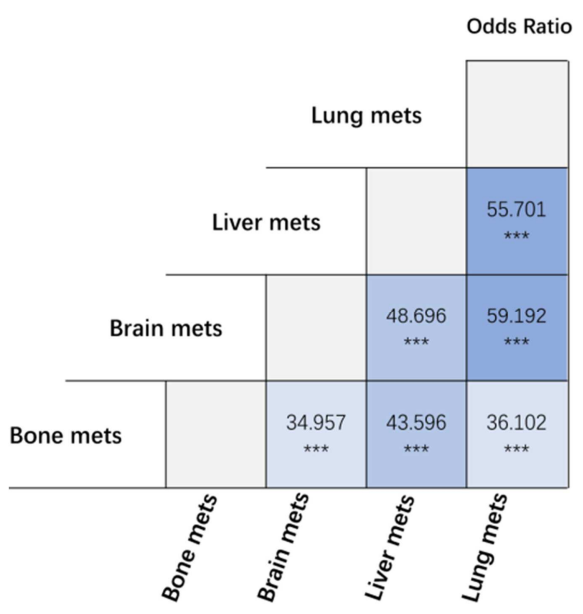

Abbreviation: mets, metastases; $* * *, \mathrm{p}<0.001$.

Figure 4. Odds ratio comparison among different bi-organ metastatic combinations. 
It is clearly shown that bladder cancer was more likely to metastasize into bone than the other three sites $(36.6 \%$, 1029/2812) (Figure 3), among which over a half were diagnosed with isolated bone metastases. Lung was the second common site for patients with bladder cancer to metastasize into $(35.1 \%, 988 / 2812)$, only next to the bone. 687 patients with bladder cancer were diagnosed with liver metastases at diagnosis (1.0\%), including 288 isolated liver metastases. Only a total of 97 patients with bladder cancer were diagnosed with brain metastases $(0.1 \%)$, with 32 isolated brain metastases, which was the least among the four metastatic sites. In addition, there were 762 patients metastasizing into other sites. Many patients were diagnosed with more than one metastatic site initially. Figure 3 showed all the possible combinations of these sites of metastasis. Bone (597/2812) and lung (526/2812) were the two most common metastatic sites as one-site metastasis. As for bi-organ metastases, the combination of bone and lung $(175 / 2812)$ was more frequent, followed by liver and lung
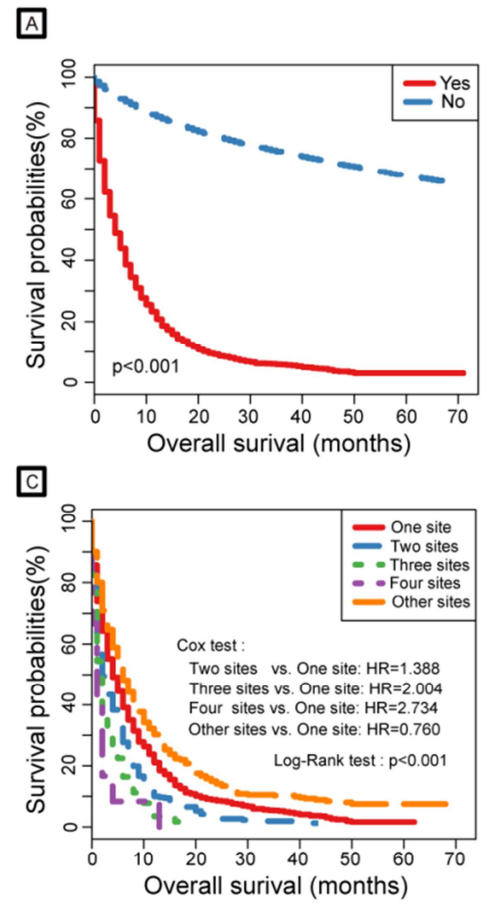

因

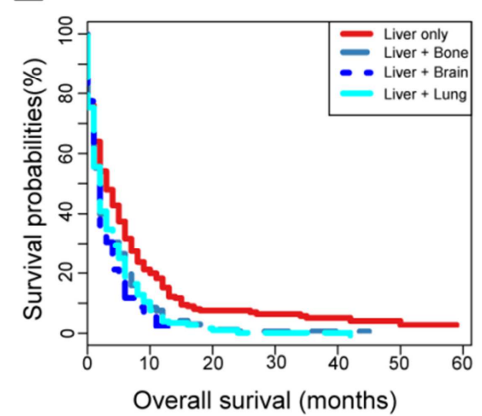

Figure 5. Kaplan-Meier curves of overall survival. metastases (144/2812), bone and liver metastases (125/2812). Moreover, in order to better understand the specific distribution of bi-organ metastases, the odds ratio of bi-organ metastasis among the four sites (bone and brain, bone and liver, bone and lung, brain and liver, brain and lung, liver and lung) was analyzed (Figure 4). In terms of three-site metastases, the bone, liver and lung combinations was predominant.

\subsection{Survival}

In our study, 24.5\% (16075/65541) deaths were observed, including 2362 patients with distant metastases at initial diagnosis. The prognosis of patients with bladder cancer and distant metastases at diagnosis was poor. The median survival time of overall survival was 4 months ( $95 \%$ CI, 3-4 months), 3 months (95\% CI, 3-4 months), 2 months (95\% CI, 2-3 months), 2 months (95\% CI, 2-4 months) with bone, lung, liver and brain metastases, respectively.

B

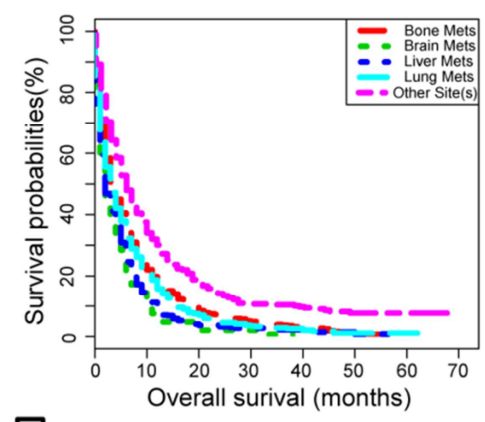

回

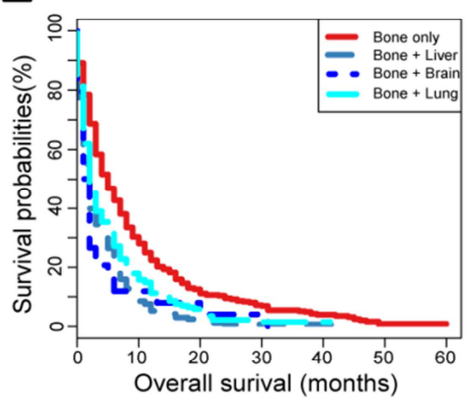

田

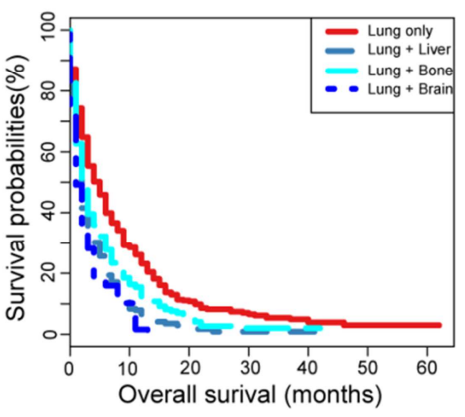

A. Kaplan-Meier curves of overall survival for bladder cancer patients with or without metastases.

B. Kaplan-Meier curves of overall survival for bladder cancer patients according to the site of distant metastases.

C. Kaplan-Meier curves of overall survival for bladder cancer patients with different number of metastatic sites.

D. E. F. Kaplan-Meier curves of overall survival comparisons in patients with differed bi-organ metastatic patterns.

Abbreviation: Mets, metastases; Other sites, having distant metastasis but the exact site was unknown; HR, hazard ratio. 
Cox regression model

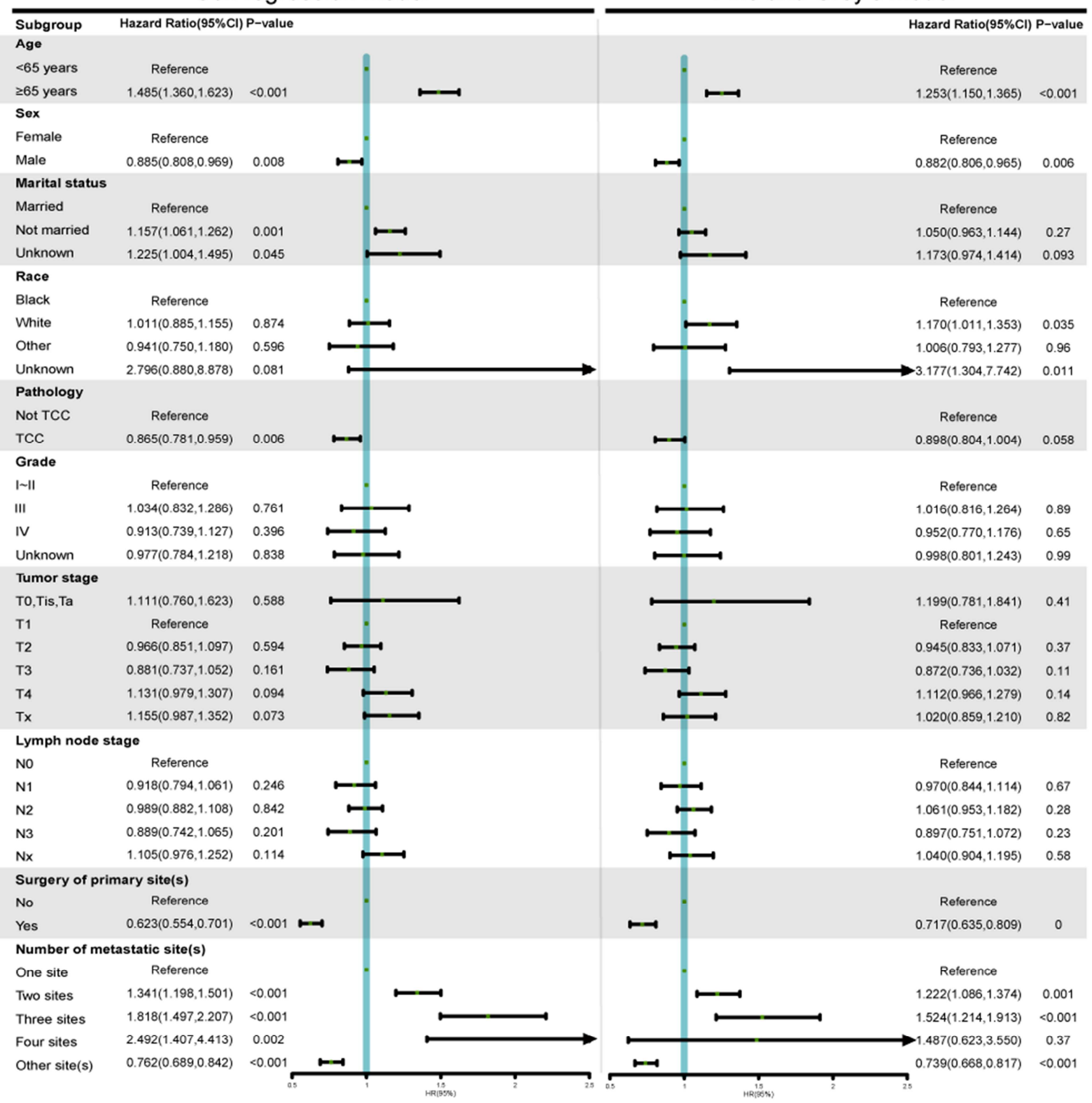

Abbreviation: CI, confidence interval; TCC, transitional cell carcinoma; Other sites, having distant metastasis but the exact site was unknown.

Figure 6. Multivariate Cox regression and Fine and Grey's model for analyzing prognostic factors for patients with bladder cancer and distant metastases at initial diagnosis.

The Kaplan- Meier survival curves of OS were showed in Figure 5. The differences of OS between patients with or without distant metastases are extraordinarily huge $(\mathrm{P}<$ 0.001) (Figure 5A). Bladder cancer patients with other sites metastases (other than bone, lung, liver and brain) had a better prognosis, while those who with brain metastases had a worse one (Figure 5B). Besides, the more sites they metastasized to, the shorter survival time they would have (univariable Cox regression analysis, $\mathrm{P}<0.001$ ) (Figure 5C). There were also differences between different bi-organ metastatic patterns (Figure 5D-F). Patients with isolated bone metastases had a better prognosis than when metastases combined with other three sites, so did isolated liver metastases and isolated lung metastases. No matter what metastatic site, once combined with brain metastases, it resulted in worse prognosis.

In order to figure out the demographic and related clinical characteristics for patients with initial bladder cancer, multivariable Cox regression and Fine and Grey's model analyses were performed (Figure 6). Age, sex, marital status, 
histology subtype, surgery and the number of distant metastatic sites were the independent prognostic factors for overall survival $(\mathrm{P}<0.05)$. On multivariable Cox regression, older age ( $\geq 65$ years vs $<65$ years; hazard ratio [HR], 1.485; 95\% CI, 1.360-1.623; $\mathrm{P}<0.001$ ), unmarried social status (vs married social status; HR, 1.157; 95\% CI, 1.061-1.262; $\mathrm{P}=0.001$ ), and metastatic disease to two sites (vs one site; HR, 1.341; 95\% CI, $1.198-1.501 ; \mathrm{P}<0.001$ ), metastatic disease to three sites (vs one site; HR, 1.818; 95\% CI, 1.497-2.207; $\mathrm{P}<0.001$ ), metastatic disease to four sites (vs one site; HR, 2.492; 95\% CI, 1.407-4.413; $\mathrm{P}=0.002$ ) were significantly associated with a poorer prognosis. Male (vs Female; HR, 0.885; 95\% CI, 0.808-0.969; $\mathrm{P}=0.008$ ), TCC subtype (vs non-TCC; HR, 0.865; $95 \% \mathrm{CI}, 0.781-0.959 ; \mathrm{P}=0.006$ ), surgery to primary tumor (vs no surgery/unknown; HR, 0.623; 95\% CI, 0.554-0.701; $\mathrm{P}<0.001)$ were significantly related to a better prognosis. The results of Fine and Grey's model analysis were almost the same as the Cox regression (Figure 6).

\section{Discussion}

In conclusion, we mainly illustrated four points in our study: (i) Concluding the distribution of distant metastases of bladder cancer. (ii) Summarizing the different metastatic combinations. (iii) Identifying risk factors of metastases. (iv) Analyzing survival rates and prognostic factors in metastatic bladder cancer. Some of the findings in metastatic bladder cancer may be valuable in making clinical decisions.

The probability of metastases for bladder cancer patients presented an age-related distribution. Specifically, younger patients $(<65$ years) were more likely to have distant metastases. We corroborate the findings of previous studies, where patients with younger age are more likely to metastasize $[8,10]$; they therefore should be imaged more extensively. Black race has always been regarded as a risk factor for many advanced cancers, and predominantly has poor prognosis due to socioeconomic status $[7,11]$. Additionally, race disparities were not included in the independent prognostic factors of bladder cancer with distant metastases at diagnosis. So great efforts and interventions should be made to improve survival outcomes in the near future. Bone, in our study, was the most common organ of metastases, followed by lung, which was not completely consistent with some of the previous researches [10, 12, 13]. It had been found that distant lymph nodes and lung were the most common sites of metastases in metastatic bladder cancer [13-15]. As for bone metastasis, it remained debatable. But Bianchi's results, which were similar to ours, revealed that high proportion of bone metastases were observed in bladder cancer patients and were even higher than lung metastases [8]. These differences might be due to the small patient cohorts previous studies contained $[3,10,12,13,16]$. Since bone and lung metastases are most common, regular imaging of these sites should be considered as a routine examination, such as computed tomography (CT), bone scintigraphy, or positron emission tomography-computed tomography (PET-CT) if necessary, discovering the distant metastases earlier. Although the incidence of brain metastases is $0.1 \%(97 / 65541)$ or even less, the prognosis is poor. Dramatic attention should be paid on brain metastases for diagnosis and treatment.

Based on our survival analysis, we found that patients with metastatic bladder cancer at initial diagnosis were connected with a dramatically higher risk of mortality when they had the following factors: older age ( $\geq 65$ years), female, unmarried social status, non-TCC subtypes, absence of surgery to the primary site and more metastatic sites. It had been proved that surgery to the primary bladder tumor could improve patients' OS outcomes in our study, so surgery to the primary site may be taken into consideration for patients who were diagnosed with bladder cancer and distant metastases when there are still surgical opportunities. But for surgery to the metastatic sites, due to the limitation of SEER database, we did not get enough information to analyze whether it could result in survival benefits for patients with bladder cancer and distant metastases at initial diagnosis. Besides, patients with one metastatic site were related to better survival when compared to those who with two, three or four metastatic sites (Figure 5C and Figure 6), which were opposite to the results of the previous study - single-site metastasis was not an independent prognostic factor of OS [3]. Maybe different selection of covariates lead to different results.

Bladder cancer is a common malignant disease in clinical. The findings in metastatic bladder cancer in our study may help to arrange thorough evaluation and then make individualized treatment plan, and maybe has a potential influence on prognosis. So prospective studies are needed for further validation.

\section{Conclusion}

The current analysis showed that the bone was the most common site for bladder cancer to metastasize into, followed by lung, liver and brain. Age, sex, marital status, histologic subtypes, the number of metastatic sites and whether undergoing surgery or not were independent prognostic factors. These findings in metastatic bladder cancer may help to arrange thorough evaluation and then make individualized treatment plan.

\section{Acknowledgements}

This work was supported by Natural Science Foundation of Zhejiang Province, China (LQ18H160014).

\section{References}

[1] Siegel RL, Miller KD. Cancer statistics, 2019. CA Cancer J Clin. 2019; 69 (1): 7-34.

[2] Lynch CF, Davila JA, Platz CE. Cancer of the urinary bladder. In: Ries LAG, Young JL, Keel GE, Eisner MP, Lin YD, Horner M-J, Eds. SEER Survival Monograph: Cancer Survival Among Adults: U.S. SEER Program, 1988-2001, Patient and Tumor Characteristics. New York: NIH Pub, 2007, pp. 181-192. 
[3] Dong F, Shen Y, Gao F, Xu T, Wang X, Zhang X, Zhong, S, Zhang, M, Chen, S, et al. Prognostic value of site-specific metastases and therapeutic roles of surgery for patients with metastatic bladder cancer: a population-based study. Cancer Manag Research. 2017; 9: 611-626.

[4] Huang P, Lan M, Peng AF, Yu QF, Chen WZ, Liu ZL, Liu JM, Huang SH. Serum calcium, alkaline phosphotase and hemoglobin as risk factors for bone metastases in bladder cancer. PloS one. 2017; 12 (9): e 0183835.

[5] Rosenberg JE, Carroll PR, Small EJ. Update on chemotherapy for advanced bladder cancer. J Urol. 2005; 174 (1): 14-20.

[6] Matos J, Mussetto I, Podesta R, Schettini D, Gandolfo N. Bladder cancer presenting with acrometastases: a different cause of foot pain. Skeletal Radiol. 2018; 47 (7): 1003-1009.

[7] Klaassen Z, DiBianco JM, Jen RP, Evans AJ, Reinstatler L, Terris MK, Madi R. Female, Black, and Unmarried Patients Are More Likely to Present With Metastatic Bladder Urothelial Carcinoma. Clin Genitourin cancer. 2016; 14 (5): e489-e492.

[8] Bianchi M, Roghmann F, Becker A, Sukumar S, Briganti A, Menon M, Karakiewicz PI, Sun, M, Noldus J, et al. Age-stratified distribution of metastatic sites in bladder cancer: A population-based analysis. Can Urol Assoc J. 2014; 8 (3-4): E148-158.

[9] Chen C, Hu LK, Chen Y, Hou JQ. The prognostic value of histological subtype in patients with metastatic bladder cancer. Oncotarget. 2017; 8 (17): 28408-28417.
[10] Sengeløv L, Kamby C, von der Maase H. Pattern of metastases in relation to characteristics of primary tumor and treatment in patients with disseminated urothelial carcinoma. Journal urol. 1996; 155 (1): 111-114.

[11] Prout GR, Wesley MN, Greenberg RS, Chen VW, Brown CC, Miller AW, Weinstein RS, Robboy SJ, Haynes MA, et al. Bladder cancer: race differences in extent of disease at diagnosis. Cancer. 2000; 89 (6): 1349-1358.

[12] Wallmeroth A, Wagner U, Moch H, Gasser TC, Sauter G, Mihatsch MJ. Patterns of metastasis in muscle-invasive bladder cancer (pT2-4): An autopsy study on 367 patients. Urol Int. 1999; 62 (2): 69-75.

[13] Shinagare AB, Ramaiya NH, Jagannathan JP, Fennessy FM, Taplin ME, Van den Abbeele AD. Metastatic pattern of bladder cancer: correlation with the characteristics of the primary tumor. AJR Am J Roentgenol. 2011; 196 (1): 117-122.

[14] Abe T, Kitamura H, Obara W, Matsumura N, Tsukamoto T, Fujioka T, Hara I, Murai S, Shinohara N, et al. Outcome of metastasectomy for urothelial carcinoma: a multi-institutional retrospective study in Japan. J Urol. 2014; 191 (4): 932-936.

[15] Otto T, Krege S, Suhr J, Rübben H. Impact of surgical resection of bladder cancer metastases refractory to systemic therapy on performance score: a phase II trial. Urology. 2001; 57 (1): 55-59.

[16] Anderson RS, el-Mahdi AM, Kuban DA, Higgins EM. Brain metastases from transitional cell carcinoma of urinary bladder. Urology. 1992; 39 (1): 17-20. 